\title{
Terapia de resincronización cardiaca en un paciente con miocardiopatía inducida por marcapasos
}

\section{Cardiac resynchronization therapy in a patient with pacemaker-induced cardiomyopathy}

\author{
María Camila luna-España, Jainer José Méndez-Flórez, José Alfredo Restrepo, \\ Andrés Buitrago-Sandoval • Bogotá, D.C. (Colombia)
}

DOI: https://doi.org/10.36104/amc.2019.1300

\begin{abstract}
Resumen
Las complicaciones derivadas de la terapia de estimulación cardiaca tienen alta prevalencia, sin embargo, a menudo son olvidadas por el personal médico. La miocardiopatía secundaria a la disincronía biventricular por la alta carga de estimulación ventricular derecha es una causa frecuente de falla cardiaca, aumentando el riesgo de muerte, hospitalizaciones y disminuyendo la calidad de vida en los pacientes con marcapasos. Presentamos un caso de una paciente que desarrolló falla cardiaca, se descartaron las causas más comunes de disfunción ventricular izquierda y se consideró secundaria a miocardiopatía inducida por marcapasos, se le realizó cambio de dispositivo por terapia de resincronización cardiaca biventricular con adecuada respuesta clínica. Resaltamos la importancia de buscar en los pacientes con falla cardiaca complicaciones asociadas a dispositivos de estimulación ventricular derecha y considerar dentro del tratamiento el cambio a estimulación biventricular. (Acta Med Colomb 2019; 44. DOI: https://doi.org/10.36104/amc.2019.1300).
\end{abstract}

Palabras clave: miocardiopatía inducida por marcapasos, falla cardiaca, terapia de resincronización cardiaca.

\begin{abstract}
There is a high prevalence of cardiac stimulation therapy complications; however, they are frequently forgotten by medical personnel. Cardiomyopathy secondary to biventricular dyssynchrony due to high right ventricular stimulation is a frequent cause of heart failure, increasing the risk of death and hospitalization and decreasing the quality of life of patients with pacemakers. We present a case of a patient who developed heart failure. The most common causes of left ventricular dysfunction were ruled out, and it was determined to be secondary to pacemaker-induced cardiomyopathy. The patient's device was changed to biventricular cardiac resynchronization therapy, with an adequate clinical response. We highlight the importance of looking for complications related to right ventricular stimulation devices in patients with heart failure, and of considering a change to biventricular stimulation within the treatment plan. (Acta Med Colomb 2019; 44. DOI: https:// doi.org/10.36104/amc.2019.1300).
\end{abstract}

Key words: Pacemaker-induced cardiomyopathy, heart failure, cardiac resynchronization therapy.
Dra. María Camila Luna-España: Residente de Medicina Interna, Universidad El Bosque; Dr. Jainer José Méndez-Flórez: Especialista en Medicina Interna, Residente Cardiología Univerversidad El Bosque; Dr. José Alfredo Restrepo: Especialista en Medicina Interna, Cardiología y Electrofisiología, Fundación Santa Fe de Bogotá; Dr. Andrés Buitrago-Sandoval: Especialista en Medicina Interna, Cardiología, Epidemiología, Master en Insuficiencia Cardíaca. Jefe Asociado de Cuidado Crítico, Fundación Santa Fe de Bogotá. Bogotá, D.C. (Colombia). Correspondencia: Dr. Jainer José MéndezFlórez. Bogotá, D.C. (Colombia)

E- mail: jainer87@hotmail.com

Recibido: 10/I/2019 Aceptado: 30/X/2019

\section{Introducción}

El síndrome de falla cardiaca continúa siendo una de las entidades más prevalentes a nivel mundial, en la actualidad hay datos que sustentan una prevalencia de $23.3 \%$, la cual aumenta de manera directamente proporcional a la edad
(1). Dentro de sus principales etiologías podemos señalar a la cardiopatía isquémica como la causa más común de falla cardiaca, sin embrago, otras patologías como la hipertensión arterial, valvulopatías, trastornos de la conducción, miocardiopatías, las enfermedades transmitidas por vectores 
y las complicaciones secundarias a dispositivos como los marcapasos, cobran gran importancia dentro de los factores de riesgo para desarrollar falla cardiaca.

El manejo médico con inhibidores de la enzima convertidora de angiotensina, los antagonistas del receptor de angiotensina, los betabloqueadores, los antagonistas del receptor de aldosterona y los inhibidores de la neprilisina han modificado el curso natural de la enfermedad, aumentando la supervivencia de los pacientes, disminuyendo el número de hospitalizaciones, mejorando la calidad de vida; a pesar de ello un número reducido de pacientes no responden a estos medicamentos y se hace necesario el implante de ciertos dispositivos como la terapia de resincronización cardiaca (CRT) logrando así mejorar los síntomas y disminuir la mortalidad en esta población (2).

Las indicaciones de la terapia de resincronización cardiaca están claramente sustentadas en las diferentes guías internacionales de falla cardiaca con un buen grado de evidencia (1). Tabla 1.

Dentro de las indicaciones dadas anteriormente las menos frecuente es la terapia de resincronización en pacientes que desarrollan falla cardiaca por estimulación eléctrica ventricular derecha; sin embargo, en algunos casos la CRT es la única alternativa terapéutica que se puede ofrecer para mejorar la fracción de eyección y la sintomatología.

Presentamos un caso de una paciente de 62 años con indicación para marcapasos bicameral por bloqueo av completo que posterior al implante del dispositivo desarrolla falla cardiaca con indicación de cambio de dispositivo a un resincronizador cardiaco, puesto que se consideró la miocardiopatía por marcapasos como la única causa de falla cardiaca en esta paciente luego de descartar otras causas más comunes como cardiopatía isquémica y miocarditis.

\section{Caso clínico}

Paciente de sexo femenino, 62 años, con antecedente de lupus eritematoso sistémico, artritis reumatoidea, bloqueo auriculo ventricular de alto grado con implante de marcapaso bicameral DDD, con estudios realizados previos al implante
Tabla 1. Indicaciones de la terapia de resincronización cardiaca.

- Pacientes en ritmo sinusal con fracción de eyección menor de 35\% sintomáticos a pesar de manejo médico óptimo por tres meses.

- Expectativa de vida mayor de un año.

- Bloqueo completo de rama izquierda con QRS mayor de 150 msegundos (IA) o entre 130 - 150 msegundos. (IB).

- Bloqueo completo de rama derecha con QRS mayor de 150 msegundos (IIA) o entre 130 - 150 msegundos. (IIB).

- Pacientes sintomáticos con fracción de eyección reducida quienes por existencia de bloqueo av de alto grado requieren estimulación ventricular. (IA).

- Pacientes con marcapasos convencional o CDI con estimulación ventricular derecha que desarrollan falla cardiaca a pesar de manejo médico óptimo. (IIB).

del dispositivo: ecocardiograma normal, resonancia magnética de corazón normal y cateterismo cardiaco sin lesiones significativas de las arterias coronarias. Presenta cuadro de 40 días de evolución caracterizado por disminución de su clase funcional, disnea de medianos a pequeños esfuerzos y edema de miembros inferiores. Al examen físico con presión arterial 140/70 mm Hg, frecuencia cardiaca 80 latidos por minuto y frecuencia respiratoria 18 respiraciones por minuto. Se observa ingurgitación yugular grado II a 45 grados, ruidos cardiacos rítmicos, sin soplos, ruidos respiratorios con estertores escasos en base. Reflujo hepatoyugular presente, sin ascitis ni hepatomegalia, con edema de extremidades inferiores grado II. Se solicitó electrocardiograma con evidencia de ritmo de marcapasos (Figura 1), BNP $4410 \mathrm{pg} / \mathrm{mL}$ y ecocardiograma transtorácico con función sistólica global disminuida (FEVI:35\%), sin alteración en la contractilidad segmentaria con ventrículo izquierdo con hipertrofia concéntrica mayor en el ápex, con estos hallazgos se hospitaliza con impresión diagnóstica de falla cardiaca con fracción de eyección reducida y se inicia manejo con bloqueo neurohumoral y diurético.

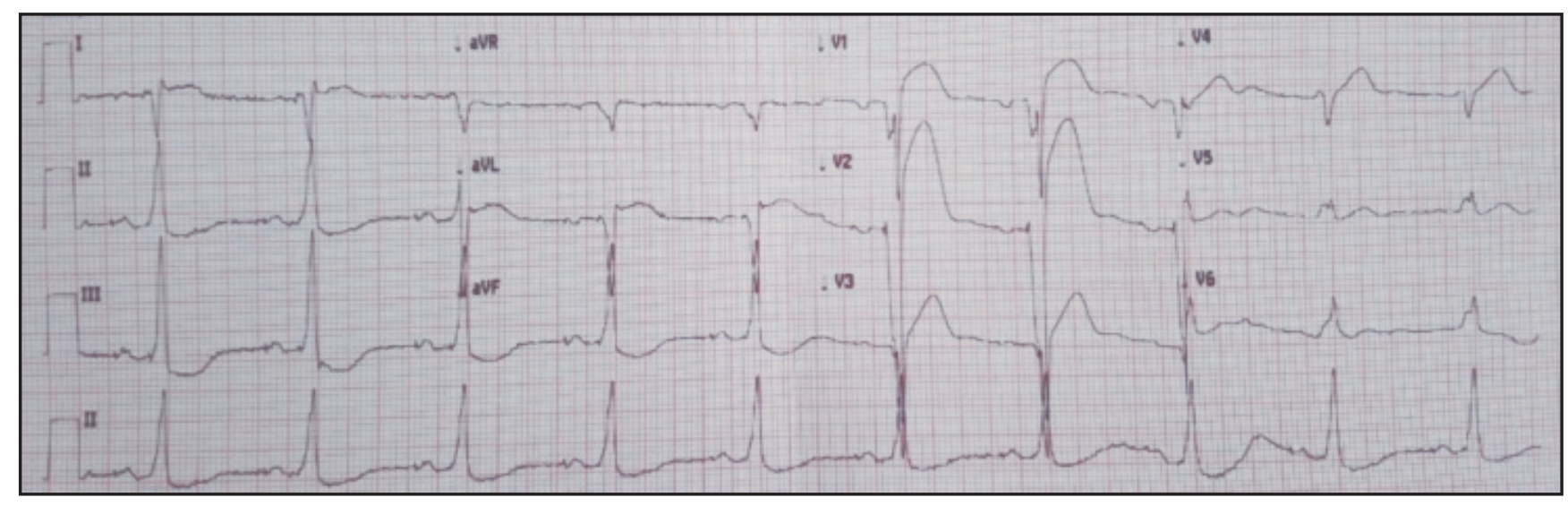

Figura 1. Electrocardiograma. Ritmo de marcapasos. Morfología de bloqueo de rama izquierda. Trastorno difuso de la repolarización. 
Por sus antecedentes fue valorada por el servicio de reumatología quienes descartaron actividad lúpica. Se realizó resonancia magnética cardiaca (Figura 2) donde no se identifica realces anormales, ni áreas de cicatrices ni infiltración sugestiva de miocarditis o isquémica, con disminución de la fracción de eyección $32 \%$ y dilatación de cavidades derechas, ante estos hallazgos se descarta etiología por isquemia, miocarditis y enfermedad reumatológica; se considera diagnóstico de miocardiopatía inducida por marcapasos. Se decide continuar tratamiento médico óptimo para falla cardiaca y realizar cambio de dispositivo de estimulación eléctrica de marcapasos convencional a cardio-resincronizador, la paciente mejora su sintomatología, se compensa de su falla cardiaca y posteriormente se realiza ecocardiograma control con fracción de eyección recuperada de $50 \%$.

\section{Discusión}

Los pacientes con estimulación ventricular derecha por marcapasos pueden desarrollar diferentes complicaciones, dentro de ellas el síndrome de marcapasos y la miocardiopatía por marcapasos. El síndrome de marcapasos es una entidad que se presenta en $20 \%$ de los pacientes y corresponde a una disociación entre la contracción auriculoventricular en dispositivos unicamerales programados en VVI la cual se corrige implantando un marcapasos bicameral con estimulación DDD (3).

La miocardiopatía por marcapasos es otra entidad frecuente, la cual se presenta con una prevalencia variable a través del tiempo del uso del dispositivo, aproximadamente $19.5 \%$ de los pacientes a los tres años presentan miocardiopatía inducida por marcapasos y es el resultado de la disincronía biventricular por alta carga de estimulación ventricular derecha ocasionando deterioro de la fracción de eyección del ventrículo izquierdo a menos de $50 \%$ con un descenso mayor de $10 \%$ del basal del paciente (4).

Nuestra paciente presentó un deterioro de la FEVI de $28 \%$ pasando de 60 - 32\%, presentando signos y síntomas de falla cardiaca a los 12 meses del implante del dispositivo lo cual va en relación con otros estudios donde se ha encontrado una asociación entre el implante del marcapasos y el desarrollo de miocardiopatía incluso en los primeros seis meses de uso.

La carga de estimulación ventricular derecha es el factor de riesgo más importante para el desarrollo de la disincronía ventricular, se ha descrito como la incidencia de muerte y hospitalizaciones por falla cardiaca puede llegar hasta $30 \%$ de los pacientes que superan $40 \%$ de estimulación ventricular derecha, nuestra paciente inicialmente requería una estimulación ventricular de sólo 2\%; sin embargo, posteriormente aumentó a $98 \%$ incrementando el riesgo de miocardiopatía por marcapasos.

El desarrollo de falla cardiaca en estos pacientes no sólo se debe a la disincronía electromecánica, sino que esta condición es el mecanismo inicial al cual se suman el disbalance entre la oferta y demanda de oxígeno en el miocardio, la alteración en la capacidad contráctil por alteraciones en

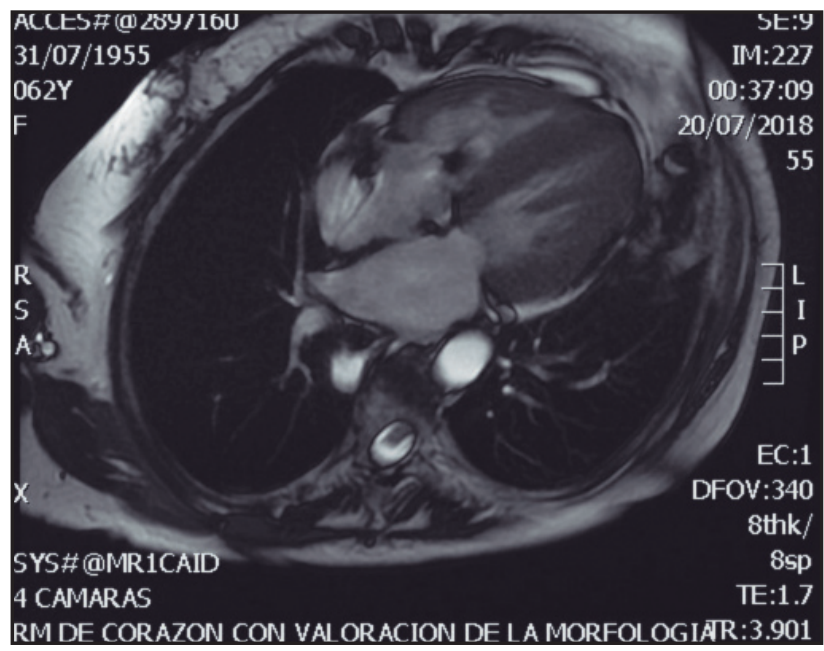

Figura 2. Resonancia magnética cardiaca. Hipertrofia concéntrica del ventrículo izquierdo. Disfunción ventricular izquierda (FEVI 32\%) sin realce tardio.

las presiones de llenado, el remodelamiento cardiaco y la activación de diferentes sistemas como el sistema nervioso simpático o el sistema renina-angiotensina-aldosterona que acentúan aún más los síntomas y signos de congestión y/o hipoperfusión (4). En nuestra paciente se documentó hipertrofia de la pared ventricular (remodelado) en el ecocardiograma y resonancia magnética que no se evidenciaba en estudios previos al implante del dispositivo, lo cual va en relación con lo descrito anteriormente; así mismo la terapia médica incluyó betabloqueadores e inhibidores de la enzima convertidora de angiotensina bloqueando los diferentes sistemas mal llamados compensadores.

Dentro de los factores de riesgo para el desarrollo de miocardiopatía por marcapasos, Khurshid y col. encontraron una relación directa con el sexo masculino (HR 2.15; $95 \%$ CI: $1.7-3.94, \mathrm{P}<.01)$ y la duración del QRS (HR 1.03 por cada un milisegundo de incremento; 95\% CI: 1.01-1.05, $\mathrm{P}<.001)(5)$. Lee y col. encontraron relación directa con la edad (HR 1.62; 95\% CI: 1.22-2.16, P < .001) (6) y Kiehl y col con el porcentaje de estímulo ventricular derecho $>20 \%$ (HR: 6.76; 95\% CI: 2.08-22.0, P <.002) (7). Otros factores de riesgo documentados por la literatura son la presencia previa de fibrilación auricular y la disminución del strain global longitudinal posterior al implante del dispositivo. En nuestra paciente el principal factor de riesgo documentado fue la estimulación cardiaca derecha, la cual alcanzó hasta $98 \%$.

Diversos mecanismos se han utilizado para tratar de prevenir la miocardiopatía por marcapasos: utilizar un sitio de estimulación alto septal a diferencia de estimulación apical no ha mostrado diferencia alguna en desenlaces duros como mortalidad y/o hospitalizaciones. Quizás las mejores estrategias que se pueden favorecer a la prevención de la miocardiopatía están la escogencia adecuada del dispositivo, el tratar de evitar al máximo la sobreestimulación ventricular y el implante inmediato de dispositivos de resincronización cardiaca en pacientes con falla cardiaca ya establecida. Cur- 
tis y col. demostraron que la estimulación biventricular en pacientes con fracción de eyección menor de 50\% disminuía la mortalidad y las hospitalizaciones por falla cardiaca al ser comparados con pacientes con sólo estimulación ventricular derecha (HR 0.74 CI 95\% 0.60-0.90) (8). Nuestra paciente no tenía deterioro previo de la fracción de eyección, por lo cual inicialmente no se consideró la estimulación biventricular pero el desarrollo de falla cardiaca si obligó a realizar el cambio de dispositivo de estimulación cardiaca.

Gage y col. demostraron como el cambio de dispositivo de marcapasos unicameral a estimulación biventricular (terapia de resincronización) se relaciona con mejoría en la fracción de eyección $(8.3 \pm 9$ vs. $5.8 \pm 9, P=0.005)$ y menor riesgo de muerte $\mathrm{u}$ hospitalizaciones por falla cardiaca (HR $0.6795 \%$ CI $0.51-0.89, P=0.005)$ (9).

Estudios en pacientes con fracción de eyección conservada también se han llevado a cabo. Man Yu y col. indicaron que a 12 meses de seguimiento el desarrollo de deterioro de la fracción de eyección era mucho menor en pacientes con estimulación biventricular que estimulación ventricular derecha (54.8\% vs. $62.2, \mathrm{P}<0.001)(10)$, estudios con mayor periodo de seguimiento también se han desarrollado, evidenciando menor descenso de la fracción de eyección y el volumen de fin de sístole a dos años con la estimulación biventricular (11). Sin embargo, es necesario tener en cuenta que los costos de la terapia de resincronización cardiaca son mucho mayores, lo cual no sería costo-efectivo en nuestra población y las complicaciones del procedimiento también son mayores en comparación con el implante del marcapasos convencional (12), quizás por estas razones no se implantó inicialmente un resincronizador en nuestra paciente.

En la paciente no se consideró esperar tres meses para determinar la respuesta terapéutica a medicamentos puesto que la causa de la falla cardiaca estaba claramente definida, considerando entonces que la miocardiopatía inducida por marcapasos podría ser una de las condiciones en las cuales el paciente podría beneficiarse de CRT sin completar tres meses de manejo médico óptimo (13).

Posterior al cambio de dispositivo se percibió en la paciente una notable mejoría clínica, la cual no se había logrado incluso con la terapia médica óptima tolerada, hasta la fecha permanece asintomática cardiovascular, sin signos clínicos de congestión o bajo gasto cardiaco y sin reingresos hospitalarios por falla cardiaca.

\section{Conclusión}

Se deben considerar las complicaciones asociadas a dispositivos de estimulación eléctrica como una de las causas frecuentes de falla cardiaca. La rápida identificación de las mismas y el reemplazo por dispositivos de estimulación biventricular mejoran los parámetros hemodinámicos como la fracción de eyección, así mismo disminuyen la sintomatología, el número de hospitalizaciones y el riesgo de muerte en estos pacientes.

\section{Referencias}

1. 2016 ESC Guidelines for the diagnosis and treatment of acute and chronic heart failure. European Heart Journal. 2016; 37: 2129-2200.

2. Cleland J, Daubert J, Erdmann E, Freemantle N, Gras D, Kappenberger L, et al. The effect of cardiac resynchronization on morbidity and mortality in heart failure. N Engl J Med 2005; 352: 1539-1549.

3. Farmer D, Estes N, Link M. New concepts in pacemaker síndrome. Indian Pacing Electrophysiol. J. 2004; 4: 195-200

4. Merchant F, Mittal S. (in press) Pacing-Induced Cardiomyopathy. Card Electrophysiol Clin 2018; DOI: https://doi.org/10.1016/j.ccep.2018.05.005

5. Khurshid S, Epstein A, Verdino R, Lin D, Goldberg L, Marchlinski F et al. Incidence and predictors of right ventricular pacing-induced cardiomyopathy. Heart Rhythm 2014; 11: 1619-25.

6. Lee SA, Cha MJ, Cho Y, Oh I, Choi E, Oh S. Paced QRS duration and myocardial scar amount: predictors of longterm outcome of right ventricular apical pacing. Heart Vessels 2016; 31: 1131-1139.

7. Kiehl EL, Makki T, Kumar R, Gumber D, Kwon D, Rickard J, et al. Incidence and predictors of right ventricular pacing-induced cardiomyopathy in patients with complete atrioventricular block and preserved left ventricular systolic function. Heart Rhythm 2016; 13: 2272-8.

8. Curtis A, Worley S, Adamson P, Chung E, Nizai I, Sherfesee L, et al. Biventricular Pacing for Atrioventricular Block and Systolic Dysfunction. N Engl J Med 2013; 368: 1585-93

9. Gage R, Burns K, Bank A. Echocardiographic and clinical response to cardiac resynchronization therapy in heart failure patients with and without previous right ventricular pacing. Eur J Heart Fail 2014; 16: 1199-1205

10. Man Yu C, Sun J, Zhang Q, Omar R, Wai G, Hussin A, et al. Biventricular Pacing in Patients with Bradycardia and Normal Ejection Fraction. N Engl J Med 2009; 361: 2123-34.

11. Sun J, Fang F, Zhang Q, Hong J, Razali O, Hussin A, et al. Biventricular pacing is superior to right ventricular pacing in bradycardia patients with preserved systolic function: 2-year results of the PACE trial. Eur Heart J 2011; 32: 2533-2540

12. Daubert J, Martins R, Leclercq C. Why We Have to Use Cardiac Resynchronization Therapy-Pacemaker More. Card Electrophysiol Clin 2015; 7: 709-720

13. Kosztin A, Vamos M, Aradi D, Schwertner W, Kovacs A, Nagy K, et al. De novo implantation vs. upgrade cardiac resynchronization therapy: a systematic review and meta-analysis. Heart Fail Rev 2018; 23: 15-26 